\title{
Application of Symbolic Manipulation to Hecke Transformations of Modular Forms in Two Variables
}

\author{
By Harvey Cohn and Jesse Deutsch*
}

Dedicated to Daniel Shanks on the occasion of his 70 th birthday

\begin{abstract}
The Hecke transformation of modular forms in several variables generates nonsymmetric modular forms out of symmetric forms. This is useful since symmetric forms arise out of Eisenstein series and are easy to construct, while nonsymmetric forms are much harder to construct. A symbolic manipulation system is required because of the magnitude of the Fourier expansions. This process is carried out for Hilbert modular functions over $\mathbb{Q}(\sqrt{2})$.
\end{abstract}

1. The Rational Case. An important application of modular functions to number theory depends on the argument multiplication in modular forms. Specifically, in the classical case, $z$ is in the upper half-plane

$$
\mathbf{H}=\{\operatorname{Im} z>0\}
$$

and modular forms are defined. These are functions $f(z)$ holomorphic in $\mathbf{H}$ which are covariant under the modular group, i.e.,

$$
f\left(\frac{a z+b}{c z+d}\right)=f(z)(c z+d)^{2 k}, \quad k \in \mathbb{Z}^{+}, a, b, c, d \in \mathbb{Z}, a d-b c=1 .
$$

Here $2 k$ is called the degree of the form. Note, because of the even exponent $2 k$, we need not worry about the identification of

$$
(a z+b) /(c z+d) \text { and }(-a z-b) /(-c z-d) .
$$

Also, $f(z)$ must approach a limit as $z \rightarrow i \infty$, and, if the limit is zero, $f(z)$ is called a cusp form. The modular (or cusp) forms of a given degree form a vector space $\mathbf{M}_{2 k}$ (or $\mathbf{C}_{2 k}$ ) of finite dimension over $\mathbb{C}$. The crux of the application is that the modular forms form a finitely generated graded ring $\mathbf{R}$. If we consider $f(n z)$ for some values of $n \in \mathbb{Z}^{+}$, then there are a set of conjugates whose symmetric functions are modular forms. We list them (for $p$ prime only):

$$
f_{\infty}(z)=p^{2 k} f(p z), \quad f_{r}(z)=f\left(\frac{z+r}{p}\right), \quad 0 \leqslant r \leqslant p-1 .
$$

Received September 26, 1985.

1980 Mathematics Subject Classification. Primary 10D20, 12 A25.

Key words and phrases. Hilbert modular functions, Hecke transformations.

*Research supported in part by NSF Grant MCS 82-01717 and by Grant \#665266 from the PSC-CUNY Research Award Program of the City University of New York. 
In particular, the following function (the Hecke-transformation):

$$
T_{p} f(z)=\sum_{j=0}^{\infty} f_{j}(z)
$$

is a modular form of degree $2 k$ again (just like $f$ ). Actually, if $f(z)$ has the Fourier expansion

$$
f(z)=a_{0}+\sum_{n=1}^{\infty} a_{n} q^{n}, \quad q=e^{2 \pi i z},
$$

then a standard calculation with roots of unity yields

$$
T_{p} f(z)=\left(p^{2 k}+p\right) a_{0}+\sum_{n=1}^{\infty}\left(p^{2 k} a_{n / p}+p a_{n p}\right),
$$

where the symbol " $a_{n / p}$ " has the value 0 when $p$ does not divide $n$.

In particular, when $f(z)$ is a cusp form, so is $T_{p} f(z)$, and if there is only one cusp form in the space $\mathbf{C}_{2 k}$,

$$
T_{p} f(z)=c_{p} f(z), \quad c_{p} \text { constant. }
$$

This is indeed true for a famous case where $2 k=12$,

$$
f(z)=q \prod_{n=1}^{\infty}\left(1-q^{n}\right)^{24}, \quad q=e^{2 \pi i z},
$$

and Mordell used this analysis to prove the multiplicative property of the coefficients $\tau(n)$ (Ramanujan's tau function) as defined by

$$
f(z)=\sum_{n=1}^{\infty} \tau(n) q^{n} .
$$

(This is the landmark achievement of Mordell [6].)

2. Modular Forms in $\mathbb{Z}[\sqrt{2}]$. Hecke provided a generalization of this procedure [5] to modular forms including those over integer rings; we take only the specific case

$$
\mathbb{Z}[\sqrt{2}]=\{a+b \sqrt{2} ; a, b \in \mathbb{Z}\} .
$$

Here the modular forms are functions of two variables, $z, z^{\prime}$ and

$$
\mathbf{H}=\left\{\operatorname{Im}(z)>0, \operatorname{Im}\left(z^{\prime}\right)>0\right\},
$$

and the modular group is [4]

$$
\Gamma:\left(z, z^{\prime}\right) \mapsto\left(\frac{\alpha z+\beta}{\gamma z+\delta}, \frac{\alpha^{\prime} z^{\prime}+\beta^{\prime}}{\gamma^{\prime} z^{\prime}+\delta^{\prime}}\right),
$$

where

$$
\alpha, \beta, \gamma, \delta \in \mathbb{Z}[\sqrt{2}], \quad \alpha \delta-\beta \gamma=1 .
$$

Here $\alpha^{\prime}=a-b \sqrt{2}$ is the conjugate over $\mathbb{Q}$ of $\alpha=a+b \sqrt{2}$. We shall define a modular form $f\left(z, z^{\prime}\right)$ of degree $2 k$ which is holomorphic and satisfies on $\mathbf{H}$

$$
f\left(\frac{\alpha z+\beta}{\gamma z+\delta}\right)=f(z)\left((\gamma z+\delta)\left(\gamma^{\prime} z^{\prime}+\delta^{\prime}\right)\right)^{2 k} .
$$

We denote $f\left(z, z^{\prime}\right)$ by $f(z)$ for brevity. Again $f(z)$ approaches a constant as $z$ (and $z^{\prime}$ ) approaches $i \infty$, and if the constant is zero, $f(z)$ is called a cusp form. As before, the definitions of the space $\mathbf{M}_{2 k}$ (or $\mathbf{C}_{2 k}$ ) of modular forms (or cusp forms) over $\mathbb{C}$ are introduced. 
For modular forms over quadratic fields (in this case $\mathbb{Q}(\sqrt{2})$ ) the more important number-theoretic applications require separate consideration of the symmetric modular group defined as

$$
\Gamma^{*}=\left\langle\Gamma, z \leftrightarrow z^{\prime}\right\rangle .
$$

For this augmented group a symmetric subspace of the forms $\mathbf{M}_{2 k}^{*}$ (or $\mathbf{C}_{2 k}^{*}$ ) is defined. In brief, it is characterized by the further property

$$
f\left(z, z^{\prime}\right)=f\left(z^{\prime}, z\right) .
$$

For $\mathbf{M}_{2 k}^{*}$ and $\mathbf{C}_{2 k}^{*}$ the symmetric modular forms and the cusp forms are generated by Eisenstein series which are intrinsically symmetric. Their expansions are of the form

$$
G_{2 k}=A_{2 k}+B_{2 k} \sum q^{b} r^{a} s_{2 k-1}(a+b \sqrt{2}), \quad k \geqslant 1,
$$

with

$$
q=e^{\pi i\left(z+z^{\prime}\right)}, \quad r=e^{\pi i\left(z-z^{\prime}\right) / \sqrt{2}}
$$

summed over some algebraic integers of $\mathbb{Z}[\sqrt{2}]$,

$$
\alpha=a+b \sqrt{2}, \quad|a|<b \sqrt{2} .
$$

Then, the coefficients are given by

$$
s_{u}(\alpha)=\sum\left|\mu \mu^{\prime}\right|^{u} \quad(\mu) \mid(\alpha),
$$

with $\mu$ summed over all ideals $(\mu)$ dividing $(\alpha)$. The condition for symmetry is $r \leftrightarrow 1 / r$, or equivalently that $s_{u}(\alpha)=s_{u}\left(-\alpha^{\prime}\right)$. We need only $k=1,2,3$ to generate the ring of symmetric modular forms of even degree. Here we use the additional constants

$$
\left(A_{2}, B_{2}\right)=(1,48), \quad\left(A_{4}, B_{4}\right)=(11,480), \quad\left(A_{6}, B_{6}\right)=(361,1008) .
$$

Actually, the more convenient generators are $\mathrm{H}_{2}, \mathrm{H}_{4}, \mathrm{H}_{6}$, given by

$$
\begin{aligned}
& H_{2}=G_{2}, \\
& H_{4}=\left(11 G_{2}^{2}-G_{4}\right) / 576, \\
& H_{6}=\frac{361 G_{2}^{3}-G_{6}-50976 G_{2} H_{4}}{224640}
\end{aligned}
$$

(see Cohn [1] and Table I for the Fourier expansions). For these forms

$$
H_{m}\left(z, z^{\prime}\right)=H_{m}\left(z^{\prime}, z\right), \quad m=2,4,6 .
$$

If we are interested in nonsymmetric positive forms of even degree, we must start with the one of lowest possible degree, 14, satisfying

$$
H_{14}\left(z, z^{\prime}\right)=-H_{14}\left(z^{\prime}, z\right) \text {. }
$$

It is arrived at (only through a very difficult analysis) by

$$
\begin{aligned}
H_{14}^{2}= & H_{4} H_{6}\left(H_{2} H_{4}+4 H_{6}\right) \\
& \times\left(H_{2}^{3} H_{6}-1728 H_{6}^{2}-288 H_{6} H_{4} H_{2}+4 H_{2}^{2} H_{4}^{2}-1024 H_{4}^{3}\right)
\end{aligned}
$$

(see Table I for the coefficients). The background material can be found in Gundlach [3] and the special form of $H_{14}$ is essentially due to Müller [7]. 


\section{TABLE I}

\section{Expansions of some modular forms}

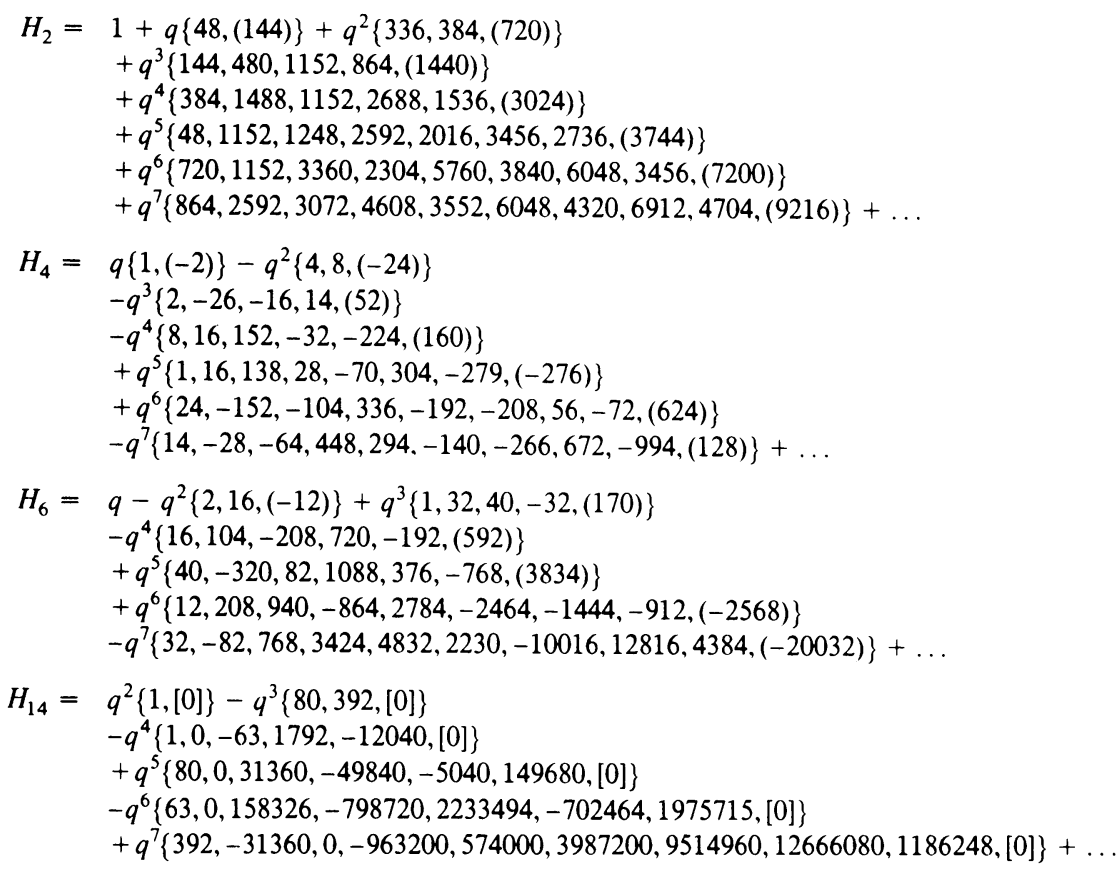

Here $\left\{a_{t}, a_{t-1}, \ldots, a_{1},\left(a_{0}\right)\right\} q^{b}$ means $\sum_{j=-t}^{\prime} a_{j} r^{j} q^{b}$ with $a_{t}=a_{-t}$ (symmetric case) and $\left\{a_{t}, a_{t-1}, \ldots, a_{1},[0]\right\} q^{b}$ means the same but with $a_{t}=-a_{t}$ (antisymmetric case).

3. The Hecke Transform in $\mathbb{Z}[\sqrt{2}]$. To construct an operator analogous to $T_{p}$ above, we take the primes in $\mathbb{Z}[\sqrt{2}]$ of degree 1 . They are given for $p=2$ by the special factorization

$$
2=\pi \pi^{\prime}, \quad \pi=2+\sqrt{2}, \quad \pi^{\prime}=2-\sqrt{2},
$$

and when $p \equiv \pm 1(\bmod 8)\left(p=A^{2}-2 B^{2}\right)$

$$
p=\pi \pi^{\prime}, \quad \pi=A+B \sqrt{2}>0, \quad \pi^{\prime}=A-B \sqrt{2}>0 .
$$

Again, we analogously define for $f\left(z, z^{\prime}\right)$ in $\mathbf{M}_{2 k}$,

$$
\begin{aligned}
& f_{\infty}\left(z^{\prime}, z^{\prime}\right)=f\left(\pi z, \pi^{\prime} z^{\prime}\right) p^{2 k}, \\
& f_{r}\left(z, z^{\prime}\right)=f\left(\frac{z+r}{\pi}, \frac{z^{\prime}+r}{\pi^{\prime}}\right), \quad 0 \leqslant r \leqslant p-1,
\end{aligned}
$$

and the Hecke operator is again seen to produce a modular form of degree $2 k$,

$$
g\left(z, z^{\prime}\right)=T_{\pi} f\left(z, z^{\prime}\right)=\sum_{0}^{\infty} f_{j}\left(z, z^{\prime}\right) .
$$


It can be shown analogously with $(1.4 \mathrm{a}, \mathrm{b})$ (see [1a]) that if

$$
f\left(z, z^{\prime}\right)=s(0)+\sum_{\alpha} s(\alpha) q^{b} r^{a}, \quad \alpha=a+b \sqrt{2},|a|<b \sqrt{2},
$$

then with $s(\beta)=0$ for $\beta \notin \mathbb{Z}[\sqrt{2}]$,

$$
T_{\pi} f\left(z, z^{\prime}\right)=\left(p^{2 k}+p\right) s(0)+\sum_{\alpha}\left(p^{2 k} s(\alpha / \pi)+p s(\pi \alpha)\right) q^{b} r^{a}
$$

Now, as even Hecke recognized, $T_{\pi}$ would destroy symmetry in general. Indeed, by the symmetry of $f\left(z, z^{\prime}\right)$,

$$
g\left(z, z^{\prime}\right)=T_{\pi} f\left(z^{\prime}, z\right)=T_{\pi^{\prime}} f\left(z, z^{\prime}\right),
$$

so $g\left(z, z^{\prime}\right) \neq g\left(z^{\prime}, z\right)$, in general. Otherwise expressed, if

$$
D_{\pi} f\left(z, z^{\prime}\right)=g\left(z, z^{\prime}\right)-g\left(z^{\prime}, z\right),
$$

then for the difference operator

$$
D_{\pi} f\left(z, z^{\prime}\right)=-D_{\pi} f\left(z^{\prime}, z\right)=-D_{\pi^{\prime}} f\left(z, z^{\prime}\right)
$$

in general, $D_{\pi} f\left(z, z^{\prime}\right) \neq 0$.

An exceptional case is for $p=2, \pi=2+\sqrt{2}$. Since $(2+\sqrt{2}) /(2-\sqrt{2})=$ $(1+\sqrt{2})^{2}$ and the operation

$$
\left(z, z^{\prime}\right) \mapsto\left(z(1-\sqrt{2})^{2}, z^{\prime}(1+\sqrt{2})^{2}\right)
$$

lies in $\Gamma$, then so does

$$
\left(z(2+\sqrt{2}), z^{\prime}(2-\sqrt{2})\right) \mapsto\left(z(2-\sqrt{2}), z^{\prime}(2+\sqrt{2})\right) .
$$

For $\pi \mid p(\neq 2)$ however, $\pi / \pi^{\prime}$ is not a unit and this operation does not have an analogue.

4. The Computation. Specifically, we shall display the transformation $T_{\pi}$ on $\mathbf{C}_{2 k}$ for $k=1,2,3,4,5,6,7$, and

$$
\begin{array}{ll}
\pi=2+\sqrt{2}, & p=2, \\
\pi=3+\sqrt{2}, & p=7,
\end{array}
$$

the two smallest primes of first order. It is unnecessary to consider $\mathbf{M}_{2 k}$ since the noncusp form $G_{2 k}$ is known to split off diagonally by

$$
T_{\pi} G_{2 k}=G_{2 k}\left(p^{2 k}+p\right)
$$

by the multiplicative nature of $s_{u}(\alpha)$. We find (see Table III) that for $\pi=2+\sqrt{2}$, $\mathrm{C}_{2 k}^{*}$ is transformed into itself, or, equivalently, is annihilated by $D_{\pi}$. On the other hand, for $\pi=3+\sqrt{2}, \mathbf{C}_{2 k}^{*}$ is not preserved but seems mapped surjectively onto $\mathrm{C}_{2 k}$. Specifically, we must go as far as $k=7$ to see this. In fact we construct $H_{14}$ from $\mathrm{C}_{2 k}^{*}$, the symmetric subspace, by means of

$$
D_{\pi}\left(H_{6}^{2} H_{2}\right)=2 \cdot 46080 H_{14}
$$

(see matrix entry in Table IV for $\pi=3+\sqrt{2}$ and $2 k=14$ ). 
TABLE II

Basis of $\mathbf{C}_{2 k}$, the cusp forms of degree $2 k$

\begin{tabular}{rll}
$2 k$ & $d$ & \multicolumn{1}{c}{$\left[B_{2 h}^{(1)}, \ldots, B_{2 h}^{(d)}\right]$} \\
\hline 4 & 1 & {$\left[H_{4}\right]$} \\
6 & 2 & {$\left[H_{6}, H_{4} H_{2}\right]$} \\
8 & 3 & {$\left[H_{6} H_{2}, H_{4}^{2}, H_{4} H_{2}^{2}\right]$} \\
10 & 4 & {$\left[H_{6} H_{4}, H_{6} H_{2}^{2}, H_{4} H_{2}^{2}, H_{4} H_{2}^{3}\right]$} \\
12 & 6 & {$\left[H_{6}^{2}, H_{6} H_{4} H_{2}, H_{6} H_{2}^{3}, H_{4}^{3}, H_{4}^{2} H_{2}^{2}, H_{4} H_{2}^{4}\right]$} \\
14 & 8 & {$\left[H_{6}^{2} H_{2}, H_{6} H_{4}^{2}, H_{6} H_{4} H_{2}^{2}, H_{6} H_{2}^{4}, H_{4}^{3} H_{2}, H_{2}^{3} H_{4}^{2}, H_{4} H_{2}^{5}, H_{14}\right]$}
\end{tabular}

TABLE III

Transformation matrix for $T_{\pi}$ on bases in Table II together with eigenvalue polynomials for $\pi=2+\sqrt{2}$

$$
\begin{array}{ccc}
2 k=4: & \underline{2 k=6}: & \left(\begin{array}{rr}
0 & 1 \\
72 & -2
\end{array}\right) \\
x+2 & x^{2}+2 x-72 \\
2 k=8: & \left.\begin{array}{ccc}
0 & 192 & 1 \\
1 & 8 & 0 \\
216 & 0 & -2
\end{array}\right) \quad \underline{2 k=10}: & \left.\begin{array}{cccc}
-24 & 0 & -2 & 0 \\
3456 & 0 & 672 & 1 \\
-96 & 1 & 8 & 0 \\
-27648 & 648 & 0 & -2
\end{array}\right) \\
x^{3}-6 x^{2}-424 x+1344 & x^{4}+18 x^{3}-1672 x^{2}-2169 x+175104
\end{array}
$$$$
\underline{2 k=12}: \quad\left(\begin{array}{cccccc}
0 & 0 & 0 & -64 & 1 & 0 \\
0 & 24 & 0 & 0 & -2 & 0 \\
0 & 13824 & 0 & -110592 & 2304 & 1 \\
-24 & -6 & 0 & -32 & 0 & 0 \\
3456 & -192 & 1 & 0 & 8 & 0 \\
-497664 & -235008 & 2232 & 0 & 0 & -2
\end{array}\right)
$$$$
(x+24)\left(x^{5}-22 x^{4}-10216 x^{3}+252736 x^{2}+18336768 x-528187392\right)
$$$$
\begin{aligned}
& 2 k=14: \quad\left(\begin{array}{cccccccc}
0 & -1152 & 0 & 0 & 32 & 1 & 0 & 0 \\
0 & 32 & 1 & 0 & 8 & 0 & 0 & 0 \\
0 & 9216 & 72 & 0 & 0 & -2 & 0 & 0 \\
0 & -3317760 & 44928 & 0 & -940032 & 8544 & 1 & 0 \\
24 & 384 & -6 & 0 & -32 & 0 & 0 & 0 \\
13824 & 0 & -288 & 1 & 0 & 8 & 0 & 0 \\
-4478976 & 15925248 & -1396224 & 8424 & 0 & 0 & -2 & 0 \\
0 & 0 & 0 & 0 & 0 & 0 & 0 & 80
\end{array}\right) \\
& (x-80)^{2}(x+112)\left(x^{5}-110 x^{4}-32552 x^{3}+3213504 x^{2}+127973376 x-4745723904\right)
\end{aligned}
$$

The method of construction is of some interest because nonsymmetric modular forms are hard to generate directly. They require a combination of such arcane devices as Poincaré series, theta series, and modular forms of half weight, while many symmetric functions are deduced effortlessly from Eisenstein series. It is necessary to have a symbolic manipulation program because the modular forms must be manipulated as Fourier series (compare Nagaoka [8]). Series with 80 terms were used routinely with some auxiliary series having 128 terms and coefficients of 
TABLE IV

Transformation matrix for $T_{\pi}$ on bases in Table II together with eigenvalue polynomials for $\pi=3+\sqrt{2}$

$$
\begin{aligned}
& \underline{2 k=4}: \quad(-8) \quad \underline{2 k=6}: \quad\left(\begin{array}{rr}
8 & -16 \\
-1152 & 40
\end{array}\right) \\
& x+8 \quad x^{2}-48 x-18112 \\
& \underline{2 k=8}: \quad\left(\begin{array}{rrr}
-616 & -1536 & 32 \\
-8 & 184 & -4 \\
6912 & -165888 & 88
\end{array}\right) \quad 2 k=10: \quad\left(\begin{array}{rrrr}
-3352 & -14 & -200 & 1 \\
-1751040 & 1064 & -113664 & 80 \\
28416 & -56 & 4552 & -4 \\
46891008 & 24192 & 940032 & 136
\end{array}\right) \\
& x^{3}+344 x^{2}-104838 x-441929216 \quad x^{4}-2400 x^{3}-83936384 x^{2}+378295830528 x-105959154151424
\end{aligned}
$$

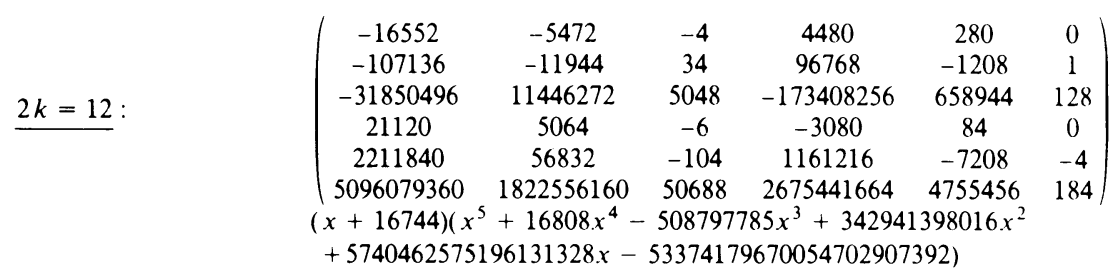

$2 k=14:$

$\left(\begin{array}{cccccccc}-35032 & 863232 & 15168 & -4 & 195712 & -1496 & 0 & 46080 \\ 4488 & 92488 & 2272 & 1 & 1920 & 66 & 0 & 768 \\ 753408 & 19998720 & 163784 & 82 & 1059840 & 7000 & 1 & -110592 \\ 573308928 & 53721169920 & 438497280 & 11336 & 410517504 & 3311616 & 176 & -382205952 \\ 7680 & -851328 & 4248 & -6 & -154904 & -12 & 0 & 18432 \\ -11059200 & 429981696 & 3513600 & -152 & 10450944 & -35096 & -4 & 3981312 \\ 137594142720 & 156959244288 & 56292212736 & 86400 & 148168507392 & 12718080 & 232 & -39749419008 \\ 1028736 & 16613376 & 96960 & -82 & 2168832 & 1864 & -1 & -74840\end{array}\right)$

$$
\begin{aligned}
& (x+175832)\left(x^{2}+149680 x-162326585792\right) \\
& \left(x^{5}-293480 x^{4}-244834408832 x^{3}+18322890100510504 x^{2}\right. \\
& \quad+9406805895241425965056 x-488479049738663380060504064)
\end{aligned}
$$

magnitude $10^{10}$. We found MACSYMA (VAX version) barely sufficient in the capacity required.

We constructed the matrix of the Hecke transformations of the basis of cusp forms of even degrees 2 to 14 and for $\pi=2+\sqrt{2}$ (which possesses symmetry) and $\pi=3+\sqrt{2}$ (which does not). The results are shown in Tables III and IV, respectively. In each case the matrices are given with respect to the bases of $\mathbf{C}_{2 k}$, namely

$$
\mathbf{C}_{2 k}=\left[B_{2 k}^{(1)}, \ldots, B_{2 k}^{(d)}\right]
$$

as shown in Table II.

The eigenvalue equations are of some theoretical interest because of their invariance under change of basis. They are of even more compelling numerical interest because the instances of rational and quadratic factors seems very difficult to fathom. (See Tables III and IV for further details.) A further study is underway by one of the authors [2]. 
Mathematics Program

City University of New York

New York, New York 10036

1. H. CoHN, "An explicit modular equation in two variables and Hilbert's twelfth problem," Math. Comp., v. 38, 1982, pp. 227-236.

1a. H. CoHN, "Cusp forms arising from Hilbert's modular functions for the field of $3^{1 / 2}$," Amer. J. Math., v. 84, 1962, pp. 283-305.

2. J. DeuTsch, Identities on Modular Forms in Several Variables Derivable from Hecke Transformations, Dissertation, Brown Univ., 1986. (In preparation.)

3. K.-B. GundlaCH, "Die Bestimmung der Funktionen zu einigen Hilbertschen Modulgruppen," $J$. Reine Angew. Math., v. 220, 1965, pp. 109-153.

4. E. HECKE, "Höhere Modulfunktionen und ihre Anwendung auf die Zahlentheorie," Math. Ann., v. 71, 1912, pp. 1-37.

5. E. HECKE, “Über Modulfunktionen und die Dirichletschen Reihen mit Eulerscher Produktentwicklung, I, II," Math. Ann., v. 114, 1937, pp. 1-28, 316-351.

6. L. J. MoRdell, “On Ramanujan's empirical expansions of modular functions," Proc. Cambridge Philos. Soc., v. 19, 1917, pp. 117-124.

7. R. MUller, “Hilbertsche Modulformen und Modulfunktionen zu Q( $\sqrt{8})$," Math. Ann., v. 266, 1983, pp. 83-103.

8. S. NAGAOKA, “On Hilbert modular forms III," Proc. Japan Acad. Ser. A Math. Sci., v. 59, 1983, pp. 346-348. 